\title{
Selección de forjados unidireccionales con criterios técnicos, económicos y sostenibles
}

\author{
Selection of one-way floor slabs based on technical, \\ economic and sustainability criteria
}

$\underline{E}_{\text {. Fraile-García }}^{(*)}$, J. Fernández-Ceniceros ${ }^{(*)}$, E. Martínez-de Pisón ${ }^{(*)}$, F.J. Martínez-de Pisón ${ }^{(*)}$

RESUMEN

En este artículo se presenta un método para la búsqueda de forjados óptimos tomando en cuenta las exigencias del Código Técnico de la Edificación, CTE, de la Instrucción de Hormigón Estructural, EHE-08, y de la buena práctica constructiva. Esta metodología se aplica al caso de forjados unidireccionales con nervio hormigonado in situ de acuerdo a las características y prestaciones habituales que ofertan las empresas del sector. Los datos de partida y las variables implicadas en el proceso hacen referencia a la viabilidad técnica, sostenibilidad y coste económico de los forjados a través de la incorporación de ratios específicos que evalúan la idoneidad de cada solución. El método aquí expuesto pretende servir de ayuda para el profesional de la construcción que necesite seleccionar aquellos forjados de coste óptimo que proporcionen el menor impacto medioambiental.

\section{SUMMARY}

This paper presents a method for finding the optimal floor slabs according to the requirements of the Spanish Building Code (CTE), the Spanish Structural Concrete Code (EHE-08) and good building practices. This method is applied to the case of one-way floor slabs with on-site cast ribs according with the characteristics and performance parameters usually offered by firms in the industry. The initial data and the variables involved in the process cover the technical viability, sustainability and monetary cost of slabs by incorporating specific ratios to assess the suitability of each solution. The method set out here may prove helpful to construction specialists who need to select optimal-cost floor slabs with the lowest environmental impacts.

Keywords: One-way floor slab; stiffness; costs; sustainability.
Palabras clave: Forjado uni
rigidez; costes; sostenibilidad.
422-31

(*) Universidad de La Rioja (España).

Persona de contacto/Corresponding author: esteban.fraile@unirioja.es (E. Fraile-García) 


\section{INTRODUCCIÓN}

\subsection{Exigencias actuales en el diseño de estructuras}

Tradicionalmente, los requerimientos exigidos en el diseño de estructuras han estado centrados en criterios de resistencia, rigidez y seguridad estructural. Pero a finales del siglo XX se incluyeron otros aspectos como la funcionalidad y durabilidad que mejoraron la calidad de las estructuras, especialmente las de hormigón armado.

En la actualidad, la cada vez más acuciante situación económica ha propiciado que los diseñadores deban esforzarse en minimizar los costes asociados a los materiales y a los procesos de fabricación y ejecución aunque, en las últimas décadas, también se ha puesto de manifiesto la necesidad de reducir el impacto medioambiental ocasionado por las edificaciones durante todo su ciclo de vida. Estas exigencias medioambientales, condicionadas por los aspectos económicos y energéticos, han dado lugar al concepto actual de sostenibilidad.

\subsection{El concepto de sostenibilidad en el diseño de estructuras}

La sostenibilidad se fundamenta en cuatro aspectos básicos que están relacionados entre sí: medio ambiente, economía, energía y sociedad.

Como dato que pueda servir para resaltar la importancia que está adquiriendo actualmente el estudio de la sostenibilidad en la construcción, sólo en Europa Occidental se estima que se emiten entre 275 y 410 millones de toneladas anuales de $\mathrm{CO}_{2}$ debidas a la producción de materiales de construcción, lo que supone entre un $8 \%$ y un $12 \%$ del total de emisiones de $\mathrm{CO}_{2}$ de esta región (1). Cabe recordar que el $\mathrm{CO}_{2}$ se considera un gas contaminante debido al efecto invernadero y que un exceso de emisiones de dicho gas puede suponer un fuerte gravamen económico para el país, lo que redundará directa o indirectamente en las empresas responsables.

De este modo, la crisis del sector de la construcción y la difícil coyuntura económica conducen a aportar un valor añadido a los productos, buscando que éstos sean competitivos en términos de coste total y que a su vez resulten sostenibles (2).

Con el objetivo de incluir estas nuevas exigencias dentro de los procesos constructivos, el Código Técnico de la Edificación (CTE) (3) ha incorporado directrices para el incremento de la calidad y sostenibili- dad de las construcciones, fomentando la innovación en los procesos constructivos y estableciendo metodologías para el control y mejora de la eficiencia energética.

De forma similar, la Instrucción de Hormigón Estructural (EHE-08) (4) recoge en su Anejo XIII un criterio para evaluar la contribución de las estructuras a la sostenibilidad a través de un indicador denominado ICES, obtenido a partir del índice de sensibilidad medioambiental de la estructura (ISMA). Su aplicación en el diseño de edificios supone tener en cuenta aspectos como la eficiencia energética o el impacto ambiental.

En este marco, las principales actuaciones que pueden realizarse son: una optimización de los diseños y recursos, la utilización de materiales ecológicos, la incorporación de materiales reciclados y la mejora del consumo energético asociado a las fases de extracción, manipulación y producción.

Además, el diseño de una estructura tendrá mayor valor a efectos de sostenibilidad cuando sea capaz de optimizar el consumo de materiales (reduciendo las cantidades de hormigón y acero) y/o cuando se incremente su vida útil, produciendo una mayor amortización de los posibles impactos desarrollados en la fase de ejecución a costa de obtener mejores aportaciones a la seguridad frente al fuego, el confort acústico, el comportamiento térmico, etc.

\subsection{Los forjados unidireccionales}

Desde hace varias décadas, los investigadores han mostrado gran interés en el estudio de los forjados unidireccionales debido a que:

1) Son los más populares en edificios de viviendas pues están presentes en el 70\% de las construcciones realizadas en España (5).

2) Tienen una relevante repercusión económica dentro del coste total de la estructura.

Dentro de los forjados unidireccionales destacan tres tipos: los forjados prefabricados de viguetas pretensadas, los de semiviguetas armadas y los de nervios hormigonados in situ.

Desde hace unos años, la utilización de estos últimos se ha visto incrementada por la generalización del sistema de encofrado total frente al tradicional de tableros y sopandas, medida adoptada para incrementar la seguridad de los operarios durante el proceso de ejecución. Esta variante proporciona una mayor flexibilidad al proyectista en cuestiones de diseño pues le ofrece la posibilidad de modificar las variables del forjado con objeto 
de buscar la solución que mejor se adapte a sus necesidades sin tener que ceñirse a las dimensiones establecidas en la "Autorización de Uso" de un determinado fabricante.

\subsection{Optimización de forjados unidireccionales}

La combinación de materiales utilizados en su construcción así como la gran variedad de soluciones técnicas posibles ha impulsado el desarrollo de algoritmos que buscan minimizar, fundamentalmente, el coste económico (6).

Aunque la optimización de estructuras de hormigón armado se viene estudiando desde hace décadas (7), estudios recientes se fundamentan en los avances del cálculo computacional utilizando técnicas de optimización avanzadas como por ejemplo la cristalización simulada o técnicas evolutivas para la búsqueda de soluciones óptimas en estructuras de hormigón (8).

En relación a los forjados de hormigón, una muestra de ello puede verse en el trabajo de Castilho et al. (9), que utilizan algoritmos genéticos modificados para obtener el diseño más económico en forjados unidireccionales con viguetas pretensadas. La función objetivo (coste económico) amplía sus límites respecto a trabajos anteriores incluyendo materias primas, costes de fabricación, costes de transporte hasta la obra y costes de ejecución.

A pesar de que en muchos de estos métodos se incluyen aspectos económicos dentro de la optimización, la ausencia de variables relacionadas con la sostenibilidad genera incertidumbre ante futuros escenarios donde sea necesario considerar estos aspectos.

En relación a trabajos de optimización encaminados a reducir emisiones de $\mathrm{CO}_{2}$ en el ámbito estructural, no existen muchas publicaciones al respecto. En este ámbito, es importante destacar el trabajo desarrollado por Payá-Zaforteza et al. (10) en el que se minimizan las emisiones de $\mathrm{CO}_{2}$ en la construcción de pórticos de edificación mediante la técnica de la cristalización simulada. Los resultados son comparados con diseños de coste óptimo observándose que ambos objetivos están relacionados y que las soluciones más comprometidas con el medio ambiente suponen un pequeño sobrecoste.

Además, actualmente se observa dentro del sector una tendencia a incorpora en la toma de decisiones aspectos relativos a otras prestaciones del forjado como la resistencia al fuego, el aislamiento acústico o el aislamiento térmico.
Estos factores resaltan la posibilidad de buscar en el proceso de diseño las mejores opciones que proporcionen además de reducciones económicas, resultados sostenibles y mejores prestaciones.

\subsection{Objetivos del trabajo presentado}

En virtud de lo anteriormente expuesto, se presenta un método de selección de forjados unidireccionales a partir de la creación de una base de datos con múltiples diseños y la búsqueda de los óptimos de Pareto que equilibren las prestaciones frente al precio. Dicha base de datos posibilita la selección de las mejores soluciones basados en criterios técnicos, económicos y sostenibles.

\section{MÉTODO}

\subsection{Definición de la geometría y materiales de cada solución}

El primer paso para poder crear la base de datos consiste en definir la geometría y los materiales a emplear de cada una de las propuestas. Las variables que definen el diseño de cada solución corresponden a la geometría de la planta (Figura 1) y la sección del forjado y materiales usados en el mismo (Figura 2).

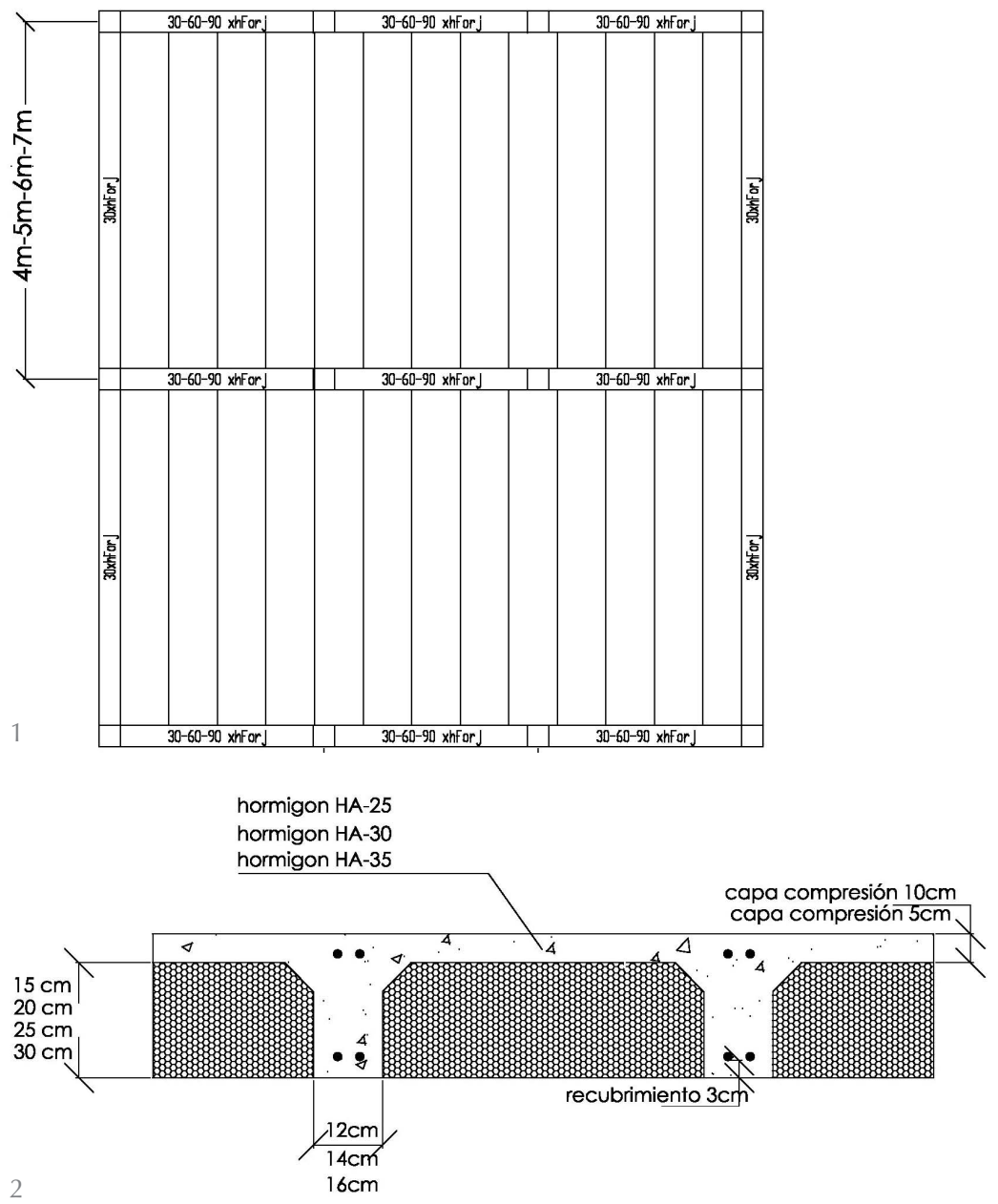

1. Plantas de forjado a estudio. 2. Secciones de forjados a estudio. 
De este modo, las variables que definen el forjado son:

- Luz del vano: que está limitada por la tipología del forjado y que, en este caso, se consideran longitudes habituales de 4, 5,6 y 7 metros.

- Dimensión de las vigas en las que apoya el forjado: para la dimensión de las vigas se eligen tres valores puntuales representativos de 30, 60 y 90 centímetros de ancho, siempre considerando el uso de vigas planas.

- Intereje: que corresponde a la suma del ancho de la bovedilla más el ancho del nervio, aunque éste solamente varía con el ancho de nervio pues la bovedilla tiene anchura constante. Para el estudio se eligen anchos de nervio de 12, 14 y 16 centímetros que dan como resultado interejes de 68, 70 y 72 centímetros, respectivamente.

- Canto total del forjado (hForj): el canto total del forjado corresponde a la suma del canto de la bovedilla y la capa de compresión. Se estudian cantos de 20, 25, 30, 35 y 40 centímetros empleando, para ello, bovedillas de 15, 20, 25 y 30 centímetros de altura con capas de compresión de 5 y 10 centímetros.

- Materiales empleados: los materiales utilizados en las bovedillas son Hormigón, Hormigón ligero y Poliestireno expandido; se han seleccionado tres resistencias características para el hormigón (25, 30 y $35 \mathrm{~N} / \mathrm{mm}^{2}$ ), así como acero B500S en todos los casos.
La combinación de las luces de vano y anchos de las vigas de apoyo proporciona los 12 casos generales que se muestran en la Tabla 1, agrupados según el ancho de las vigas.

Combinando las variables correspondientes a la sección del forjado se obtienen, para cada tipo de material de bovedilla, 72 definiciones de forjado (Tabla 2). Al ser tres materiales diferentes el número de soluciones es de $3 \times 72=216$ para cada una de las 12 definiciones recogidas en la Tabla 1, lo que supone un total de $12 \times 216=2.592$ tipos de forjados planos con cantos comprendidos entre 20 y $40 \mathrm{~cm}$.

Con objeto de simplificar el proceso, dentro del universo de posibles alternativas, no se han contemplado los casos formados por un vano interior o un vano aislado, pues se considera que el vano extremo es el más representativo en edificación residencial en cuanto a consumos y prestaciones del forjado. Habitualmente, este vano extremo es el que condiciona las prestaciones en cuanto a flecha admisible para esta tipología estructural donde las configuraciones más usadas son de dos o tres vanos.

\subsection{Cálculo de cada solución}

Una vez definido el diseño del forjado, el siguiente paso consiste en incluir las cargas contempladas en edificación. En este caso se considera una "sobrecarga de uso" (SCU) de 2,0 kN/m² y unas "cargas perma-

Tabla 1. Descripción de los 12 casos simplificados para estudio.

\begin{tabular}{|c|c|c|c|c|c|c|}
\hline \multirow{2}{*}{$\begin{array}{l}\text { Luz de } \\
\text { cálculo } \\
\quad(\mathrm{m})\end{array}$} & $\begin{array}{l}\text { Vigas } \\
0,30 \mathrm{~m}\end{array}$ & \multirow{2}{*}{$\begin{array}{c}\text { Porcentaje de superficie } \\
\text { que corresponde } \\
\text { al nervio y bovedillas }\end{array}$} & $\begin{array}{l}\text { Vigas } \\
0,60 \mathrm{~m}\end{array}$ & \multirow{2}{*}{$\begin{array}{c}\text { Porcentaje de superficie } \\
\text { que corresponde } \\
\text { al nervio y bovedillas }\end{array}$} & $\begin{array}{c}\text { Vigas } \\
0,90 \mathrm{~m}\end{array}$ & \multirow{2}{*}{$\begin{array}{c}\text { Porcentaje de superficie } \\
\text { que corresponde } \\
\text { al nervio y bovedillas }\end{array}$} \\
\hline & Long vigueta & & Long vigueta & & Long vigueta & \\
\hline 4 & 3,70 & $83,40 \%$ & 3,40 & $73,97 \%$ & 3,10 & $65,17 \%$ \\
\hline 5 & 4,70 & $85,37 \%$ & 4,40 & $77,66 \%$ & 4,10 & $70,38 \%$ \\
\hline 6 & 5,70 & $86,70 \%$ & 5,40 & $80,18 \%$ & 5,10 & $73,97 \%$ \\
\hline 7 & 6,70 & $87,66 \%$ & 6,40 & $82,02 \%$ & 6,10 & $76,60 \%$ \\
\hline
\end{tabular}

\subsection{Número de soluciones a estudiar}

La buena práctica constructiva sugiere el uso de vigas planas debido fundamentalmente a razones "económicas" de facilidad constructiva por empleo de sistemas estándar de encofrado total.

En un primer paso, se define el canto mínimo del forjado y, a partir de ese valor, se seleccionan las siguientes variables geométricas: capa de compresión, ancho de nervio, materiales a emplear y dimensiones de los apoyos (sección de las vigas). Con estos datos es posible cubrir una gran parte de los casos comúnmente utilizados en la construcción de edificaciones.
Tabla 2. Forjados con bovedillas de Hormigón ligero, Hormigón y Poliestireno expandido.

\begin{tabular}{|c|c|c|c|}
\hline $\begin{array}{l}\text { Canto de } \\
\text { bovedilla } \\
\text { (cm) }\end{array}$ & $\begin{array}{c}\text { Nervio } \\
(\mathrm{cm})\end{array}$ & $\mathrm{HA}\left(\mathrm{f}_{\mathrm{ck}}\right)$ & $\begin{array}{c}\text { Capa } \\
\text { compresión } \\
(\mathrm{cm})\end{array}$ \\
\hline \multirow{13}{*}{$15-20-25-30$} & \multirow{4}{*}{12} & 25 & $\begin{array}{r}5 \\
10\end{array}$ \\
\hline & & 30 & $\begin{array}{r}5 \\
10\end{array}$ \\
\hline & & \multirow{2}{*}{35} & $\frac{10}{5}$ \\
\hline & & & 10 \\
\hline & \multirow{5}{*}{14} & \multirow{2}{*}{25} & 5 \\
\hline & & & $\frac{10}{5}$ \\
\hline & & 30 & 10 \\
\hline & & \multirow{2}{*}{35} & 5 \\
\hline & & & 10 \\
\hline & \multirow{4}{*}{16} & 25 & 5 \\
\hline & & \multirow[b]{2}{*}{30} & $\frac{10}{5}$ \\
\hline & & & 10 \\
\hline & & 35 & $\begin{array}{r}5 \\
10\end{array}$ \\
\hline
\end{tabular}


nentes" (CM) de 2,5 kN/m² que representan las cargas del pavimento $\left(1,5 \mathrm{kN} / \mathrm{m}^{2}\right)$ más las de la tabiquería $\left(1 \mathrm{kN} / \mathrm{m}^{2}\right)$.

Después, se plantean las hipótesis de cálculo tanto para los "Estados Límite Últimos" (ELU) como para los "Estados Límite de Servicio" (ELS).

Una vez definidas las dimensiones, materiales, cargas e hipótesis de cálculo, el siguiente paso consiste en calcular las $2.592 \mathrm{com}$ binaciones de casos mediante un programa profesional de cálculo de estructuras de hormigón (11) que toma en cuenta el caso habitual de ejecución de obra, donde primero se construye la tabiquería y luego el solado.

La utilización del cálculo matricial 3D, que aporta esta herramienta informática, permite identificar cómo afecta la rigidez de los apoyos en las prestaciones del forjado. Para ello, se emplea la configuración estándar del programa que se basa en la aplicación de la EHE a partir del siguiente histórico de cargas:

1) Carga debida al peso propio: a partir del primer mes.
2) Carga debida a la tabiquería: a partir del segundo mes.

3) Carga debida al pavimento: a partir del tercer mes.

4) Entrada en servicio de la sobrecarga del uso: a partir del año.

Para cada solución se obtienen, como variables de salida, el valor de la flecha que permite estimar el grado de bondad de la solución a efectos de rigidez y los consumos de los materiales.

Los valores relativos al coste de los materiales (acero, hormigón y elementos aligerantes) así como el coste de colocación de los mismos, se obtienen del banco BEDEC del Instituto de Tecnología de la Construcción de Cataluña (ITeC) (12) (Tabla 3).

Partiendo de los costes unitarios conocidos y de los ratios de consumos de cada solución ( $\mathrm{kg}$ de Acero $/ \mathrm{m}^{2}$, litros de Hormigón $/ \mathrm{m}^{2}$ y $\mathrm{ml}$ de aligerante $/ \mathrm{m}^{2}$ ), se define el coste en $€ / \mathrm{m}^{2}$ del forjado excluyendo, con objeto de simplificar el problema, el coste del encofrado que se supone constante una vez determinado el canto y las cuantías de acero en vigas y pilares.

Tabla 3. Coste de los materiales (incluyendo colocación del acero, bovedillas y hormigón), coste energético y emisiones de $\mathrm{CO}_{2}$

\begin{tabular}{|c|c|c|c|c|}
\hline Material & Descripción & Coste & Coste energético (kwh) & Emisión $\mathrm{CO}_{2}(\mathrm{Kg})$ \\
\hline \multirow{3}{*}{ Hormigón } & $\begin{array}{c}\mathrm{m}^{3} \mathrm{HA}-25 / \mathrm{B} / 20 / \mathrm{l} \text {, de consistencia } \\
\text { blanda y tamaño máximo de árido } 20 \mathrm{~mm} \text {, } \\
\text { vertido con bomba }\end{array}$ & $93,03 € / \mathrm{m}^{3}$ & 389,88 & 260,4 \\
\hline & $\begin{array}{c}\mathrm{m}^{3} \mathrm{HA}-30 / \mathrm{B} / 20 / \mathrm{ll} \text {, de consistencia } \\
\text { blanda y tamaño máximo de árido } 20 \mathrm{~mm} \text {, } \\
\text { colocado con bomba }\end{array}$ & $102,92 € / \mathrm{m}^{3}$ & 442,82 & 303,7 \\
\hline & $\begin{array}{c}\mathrm{m}^{3} \mathrm{HA}-35 / \mathrm{B} / 20 / \mathrm{Illa} \text {, de consistencia } \\
\text { blanda y tamaño máximo de árido } 20 \mathrm{~mm}, \\
\text { colocado con bomba }\end{array}$ & $106,30 € / \mathrm{m}^{3}$ & 444,53 & 304 \\
\hline Acero & $\begin{array}{c}\text { kg Armadura para forjado unidireccional AP 500 SD } \\
\text { de acero en barras corrugadas B } 500 \text { SD } \\
\text { de límite elástico }>=500 \mathrm{~N} / \mathrm{mm}^{2}\end{array}$ & $1,20 € / \mathrm{kg}$ & 10,51 & 3,05 \\
\hline \multirow{4}{*}{$\begin{array}{l}\text { Bovedillas } \\
\text { poliestireno } \\
\text { expandido }\end{array}$} & $\begin{array}{c}\text { m de bovedilla para forjado } \\
\text { de poliestireno expandido } \\
\text { de } 56 \times 100 \mathrm{~cm} \text { y } 15 \mathrm{~cm} \text { de altura }\end{array}$ & $3,03 € / \mathrm{m}$ & 27,28 & 14,50 \\
\hline & $\begin{array}{l}\text { m de bovedilla para forjado } \\
\text { de poliestireno expandido } \\
\text { de } 56 \times 100 \mathrm{~cm} \text { y } 20 \mathrm{~cm} \text { de altura }\end{array}$ & $3,92 € / \mathrm{m}$ & 36,37 & 19,33 \\
\hline & $\begin{array}{c}\text { m de bovedilla para forjado } \\
\text { de poliestireno expandido } \\
\text { de } 56 \times 100 \mathrm{~cm} \text { y } 25 \mathrm{~cm} \text { de altura }\end{array}$ & $4,81 € / \mathrm{m}$ & 45,46 & 24,16 \\
\hline & $\begin{array}{l}\text { m de bovedilla para forjado } \\
\text { de poliestireno expandido } \\
\text { de } 56 \times 100 \mathrm{~cm} \text { y } 30 \mathrm{~cm} \text { de altura }\end{array}$ & $5,70 € / \mathrm{m}$ & 54,55 & 28,99 \\
\hline \multirow{4}{*}{$\begin{array}{l}\text { Bovedillas } \\
\text { hormigón }\end{array}$} & $\begin{array}{c}\text { m de bovedilla de Hormigón para } \\
\text { forjado de } 56 \times 20 \mathrm{~cm} \text { y } 15 \mathrm{~cm} \text { de altura }\end{array}$ & $3,17 € / \mathrm{m}$ & 49,95 & 16,82 \\
\hline & $\begin{array}{l}\text { m de bovedilla de Hormigón para } \\
\text { forjado de } 56 \times 20 \mathrm{~cm} \text { y } 20 \mathrm{~cm} \text { de altura }\end{array}$ & $3,61 € / \mathrm{m}$ & 57,46 & 19,35 \\
\hline & $\begin{array}{l}\mathrm{m} \text { de bovedilla de Hormigón para } \\
\text { forjado de } 56 \times 20 \mathrm{~cm} \text { y } 25 \mathrm{~cm} \text { de altura }\end{array}$ & $4,05 € / \mathrm{m}$ & 64,97 & 21,88 \\
\hline & $\begin{array}{l}\text { m de bovedilla de Hormigón para } \\
\text { forjado de } 56 \times 20 \mathrm{~cm} \text { y } 30 \mathrm{~cm} \text { de altura }\end{array}$ & $4,49 € / \mathrm{m}$ & 72,48 & 24,41 \\
\hline \multirow{4}{*}{$\begin{array}{l}\text { Bovedillas } \\
\text { hormigón } \\
\text { ligero }\end{array}$} & $\begin{array}{l}\mathrm{m} \text { de bovedilla de Hormigón ligero para } \\
\text { forjado de } 56 \times 20 \mathrm{~cm} \text { y } 15 \mathrm{~cm} \text { de altura }\end{array}$ & $4,95 € / \mathrm{m}$ & 34,72 & 11,99 \\
\hline & $\begin{array}{l}\mathrm{m} \text { de bovedilla de Hormigón ligero para } \\
\text { forjado de } 56 \times 20 \mathrm{~cm} \text { y } 20 \mathrm{~cm} \text { de altura }\end{array}$ & $5,66 € / \mathrm{m}$ & 39,93 & 13,79 \\
\hline & $\begin{array}{l}m \text { de bovedilla de Hormigón ligero para } \\
\text { forjado de } 56 \times 20 \mathrm{~cm} \text { y } 25 \mathrm{~cm} \text { de altura }\end{array}$ & $6,36 € / \mathrm{m}$ & 45,14 & 15,59 \\
\hline & $\begin{array}{l}\text { m de bovedilla de Hormigón ligero para } \\
\text { forjado de } 56 \times 20 \mathrm{~cm} \text { y } 30 \mathrm{~cm} \text { de altura }\end{array}$ & $7,07 € / \mathrm{m}$ & 50,35 & 17,39 \\
\hline
\end{tabular}


Además, para valorar la repercusión ambiental de cada solución, se definen dos nuevas variables: el coste energético y las emisiones de $\mathrm{CO}_{2}$ relativas a la fabricación, transporte y colocación de los tres materiales empleados: acero, hormigón y aligerantes. Estos valores se obtienen igualmente del banco BEDEC del ITeC (Tabla 3). Los costes energéticos se consideran incluidos y valorados en el coste del material.

La repercusión de las emisiones de $\mathrm{CO}_{2}$ generadas por cada una de las soluciones se establece en $20 €$ por tonelada de $\mathrm{CO}_{2}$ emitida (13), aportando un valor que sumado a su precio de coste material de ejecución nos proporciona una variable, denominada ecoprecio, que se define como:

$$
\begin{gathered}
\text { eco }{ }_{\text {precio }}=\text { Coste_material }\left(€ / \mathrm{m}^{2}\right)+ \\
+ \text { Coste_de_CO }{ }_{2} \text { emitido }\left(€ / \mathrm{m}^{2}\right)
\end{gathered}
$$

Finalmente, y con objeto de poder valorar la idoneidad técnica y económica de las diferentes alternativas, se define un parámetro que define la "rigidez" y que corresponde con el margen existente entre la flecha estimada de cada solución y la flecha admisible permitida por la norma (4 y 14). Es decir:

Rigidez $($ margen de flecha en $\mathrm{cm}$ ) = $=$ Flecha $_{\text {máxima admitida }}$ - Flecha estimada

Este parámetro de rigidez, así definido, permite valorar las prestaciones del forjado.

Como es lógico, solamente se seleccionan aquellas soluciones que obtienen un valor de rigidez positivo y que, además, cumplen con las limitaciones que establece la norma: que la solución tenga una flecha activa, en $\mathrm{cm}$, menor que L/500 y L/1000+0,5 cm; y que la flecha total, en $\mathrm{cm}$, sea menor que $\mathrm{L} / 250$ y L/500+1 cm, siendo L la longitud del vano en $\mathrm{cm}$.

\subsection{Creación de la base de datos}

Una vez calculadas todas las soluciones y seleccionadas aquellas consideradas como válidas, se genera la base de datos final donde cada solución se codifica de la siguiente manera:

[3] Mn1+n2 n3 HA-n4 rn5 Zn6

siendo:

- $\mathrm{M}$ el material de las bovedillas $(\mathrm{A}=$ Hormigón ligero, $\mathrm{H}=$ Hormigón, $\mathrm{P}=$ Poliestireno expandido).

- $n 1$ la altura de la bovedilla en cm (15, 20, 25 ó 30).
- $n 2$ la altura de la capa de compresión en cm (5 o 10).

- $n 3$ el intereje en $\mathrm{cm}$ que viene marcado por el ancho de nervio (68 para un nervio de $12 \mathrm{~cm}, 70$ para un nervio de $14 \mathrm{~cm}$ o 72 para un nervio de $16 \mathrm{~cm}$ ).

- HA seguida de n4 que identifica las prestaciones del hormigón empleado (HA-25, HA-30 o HA-35).

- $r$ seguida de $n 5$ que identifica el recubrimiento en $\mathrm{mm}$ de las armaduras empleadas, en este caso se mantiene fijo a $30 \mathrm{~mm}$.

- Z seguida de $n 6$ que identifica el ancho de las vigas de apoyo del forjado en $\mathrm{cm}$ (Z30, Z60, Z90)

Por ejemplo, la siguiente codificación: A15+5 72 HA-35 r30 Z90, corresponde a un forjado de hormigón ligero de canto total $20 \mathrm{~cm}$, con un intereje de $72 \mathrm{~cm}$, elaborado con hormigón armado de $35 \mathrm{~N} / \mathrm{mm}^{2}$, con un recubrimiento de $30 \mathrm{~mm}$ y apoyado sobre vigas de un ancho de $90 \mathrm{~cm}$.

\subsection{Interpretación de los resultados}

Una vez fijados el canto y luz del forjado por el proyectista o los requerimientos del cliente, el método permite valorar el coste y las prestaciones de las mejores soluciones de forjado combinadas con las diferentes alternativas de diseño. Para ello, cada solución se representa como un punto dentro de un gráfico donde el eje de las $Y$ representa el precio o ecoprecio, y el eje de las $X$ el valor de la Rigidez o margen de la flecha en $\mathrm{cm}$. Las soluciones se presentan ordenadas según la Rigidez de menor a mayor (de izquierda a derecha).

En primer lugar, para reducir el número de casos posibles, se seleccionan los óptimos de Pareto de las soluciones obtenidas según el tipo de material y canto. Por ejemplo, en la Figura 3 se muestran los óptimos de Pareto para los forjados de $5 \mathrm{~m}$ de luz y $30 \mathrm{~cm}$ de canto como triángulos oscuros que indican aquellas soluciones que son mejores por precio o por prestaciones (Rigidez) frente a las demás (triángulos claros).

Se denomina óptimo de Pareto a aquella solución en el que ninguna de las variables que la definen puede mejorar su situación sin reducir el valor de las otras. Por ejemplo, la solución 31 constituye un óptimo de Pareto pues se obtiene una solución más barata y con mejores prestaciones que las soluciones 28, 24, 26, 20, 19, 16, etc.; que corresponden con todas las soluciones existentes entre la 31 y la 13. En este caso, la solución 13 es otro óptimo de Pareto pues se obtiene una solución más económica que la 31 aunque con peores prestaciones. 

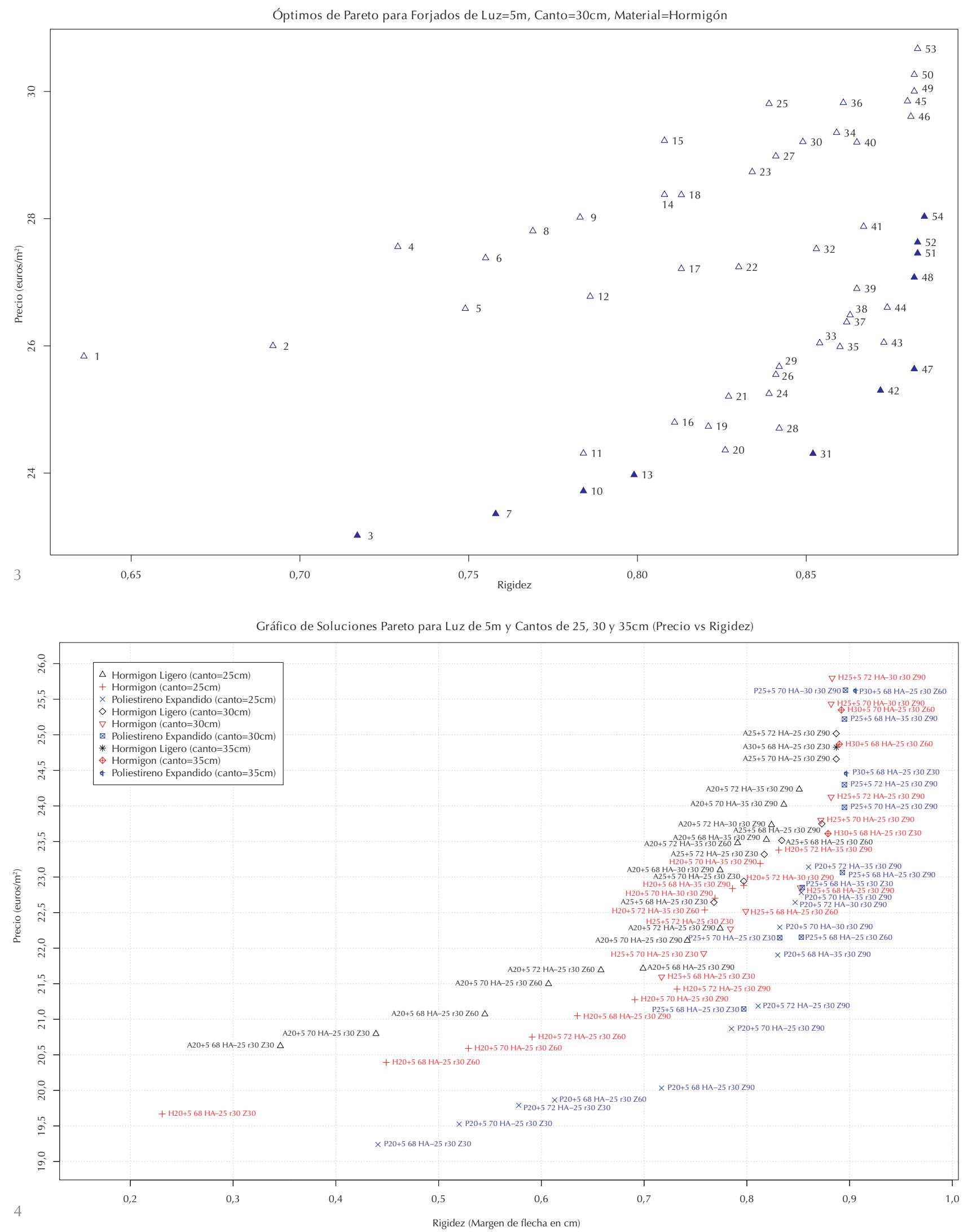

Una vez obtenidos todos los óptimos de Pareto, se representan juntos en una gráfica como las de las Figuras 4 y 5, donde pueden compararse las mejores soluciones según el "coste" frente a la Rigidez de la solución y para todos los tipos de material y cantos considerados.
Las mejores soluciones, para los tres materiales de bovedillas, se sitúan en el cuadrante inferior derecho donde existen altos valores de rigidez y bajos "costes" por metro cuadrado. De entre ellas, el diseñador puede seleccionar aquella que mejor se adapte a su proyecto sabiendo que está eli-
3. Optimos de Pareto para forjados de luz $=5 \mathrm{~m}$, canto $=30 \mathrm{~cm}$ y bovedilla de Hormigón.

4. Óptimos de Pareto para forjados de luz $=5 \mathrm{~m}$ (precio frente a rigidez). 
5. Óptimos de Pareto para forjados de luz $=5 \mathrm{~m}$ (ecoprecio frente a rigidez).

6. Óptimos de Pareto para forjados de luz $=6 \mathrm{~m}$ (precio frente a rigidez). giendo entre las soluciones que cumplen los mejores requerimientos. La decisión final dependerá del valor de rigidez que estime oportuno en relación con el coste del forjado y el tipo de material a utilizar.
A continuación, se muestran los resultados para forjados con luces de 5 y 6 metros, $y$ cantos de 25, 30 y 35 centímetros; que son de los más frecuentes en edificación (Figuras $4,5,6$ y 7 ).

Gráfico de Soluciones Pareto para Luz de 5m y Cantos de 25, 30 y 35cm (Eco_Precio vs Rigidez)

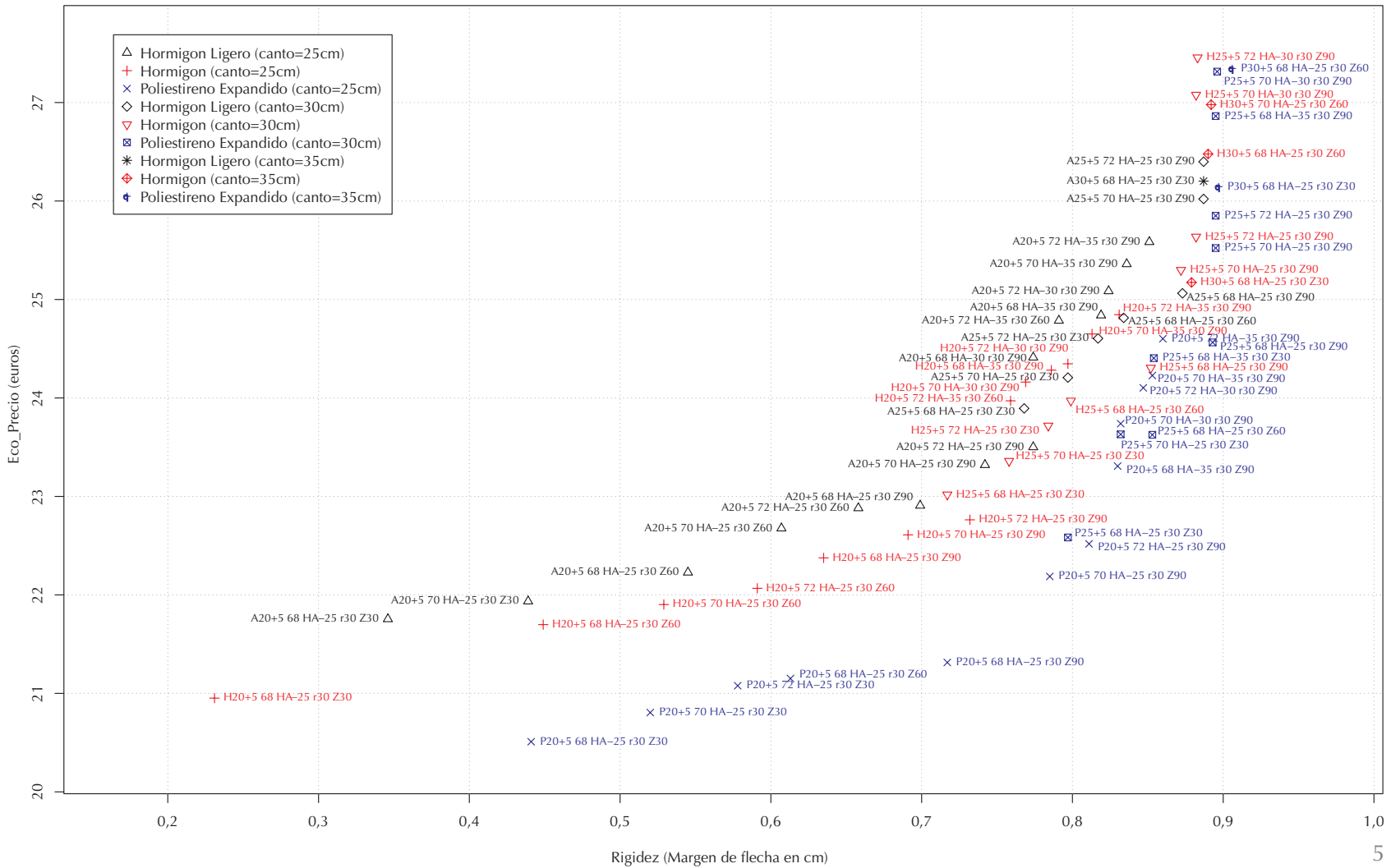

Gráfico de Soluciones Pareto para Luz de 6m y Cantos de 25, 30 y 35cm (Precio vs Rigidez)

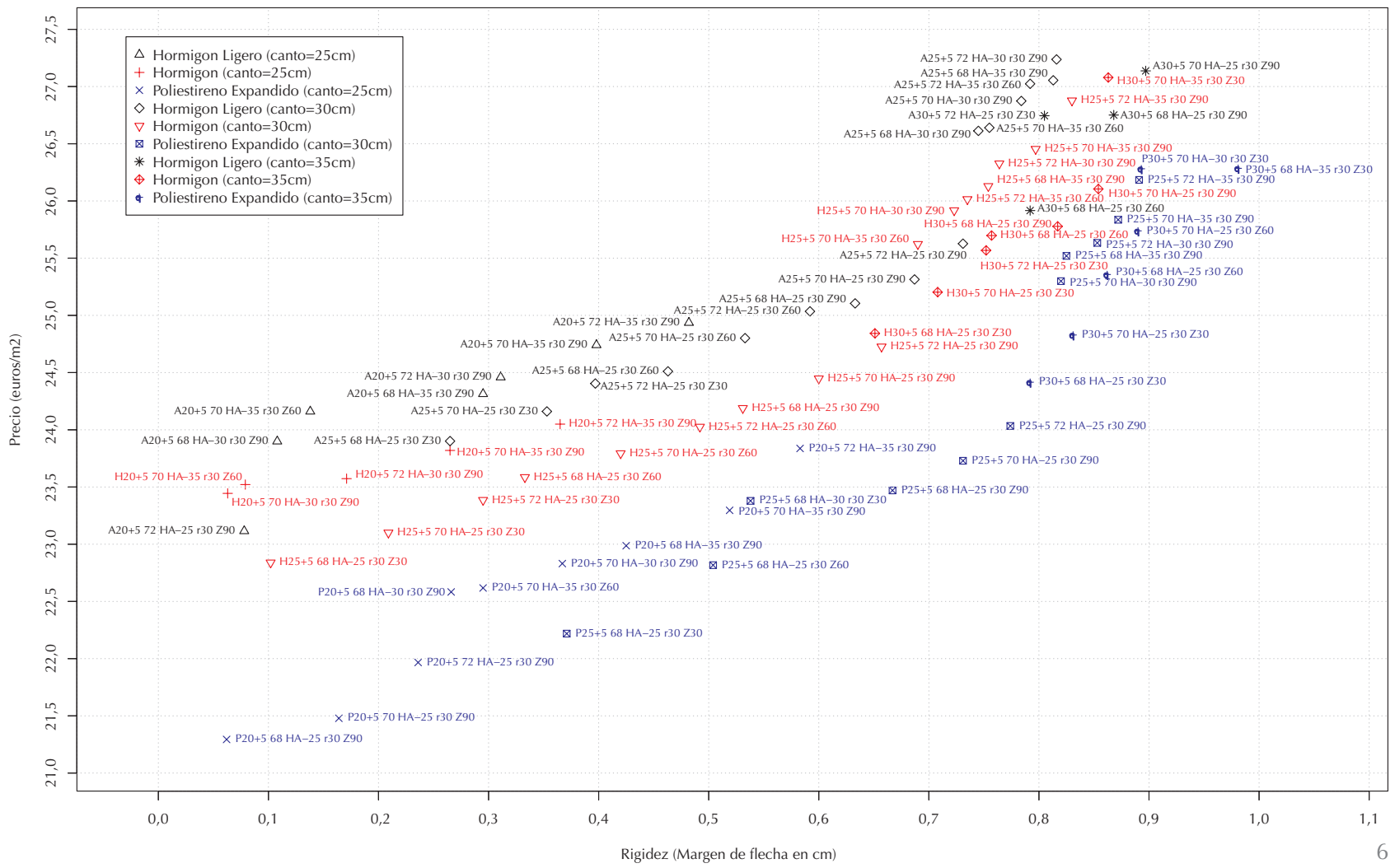


Gráfico de Soluciones Pareto para Luz de 6m y Cantos de 25, 30 y 35cm (Eco_Precio vs Rigidez)

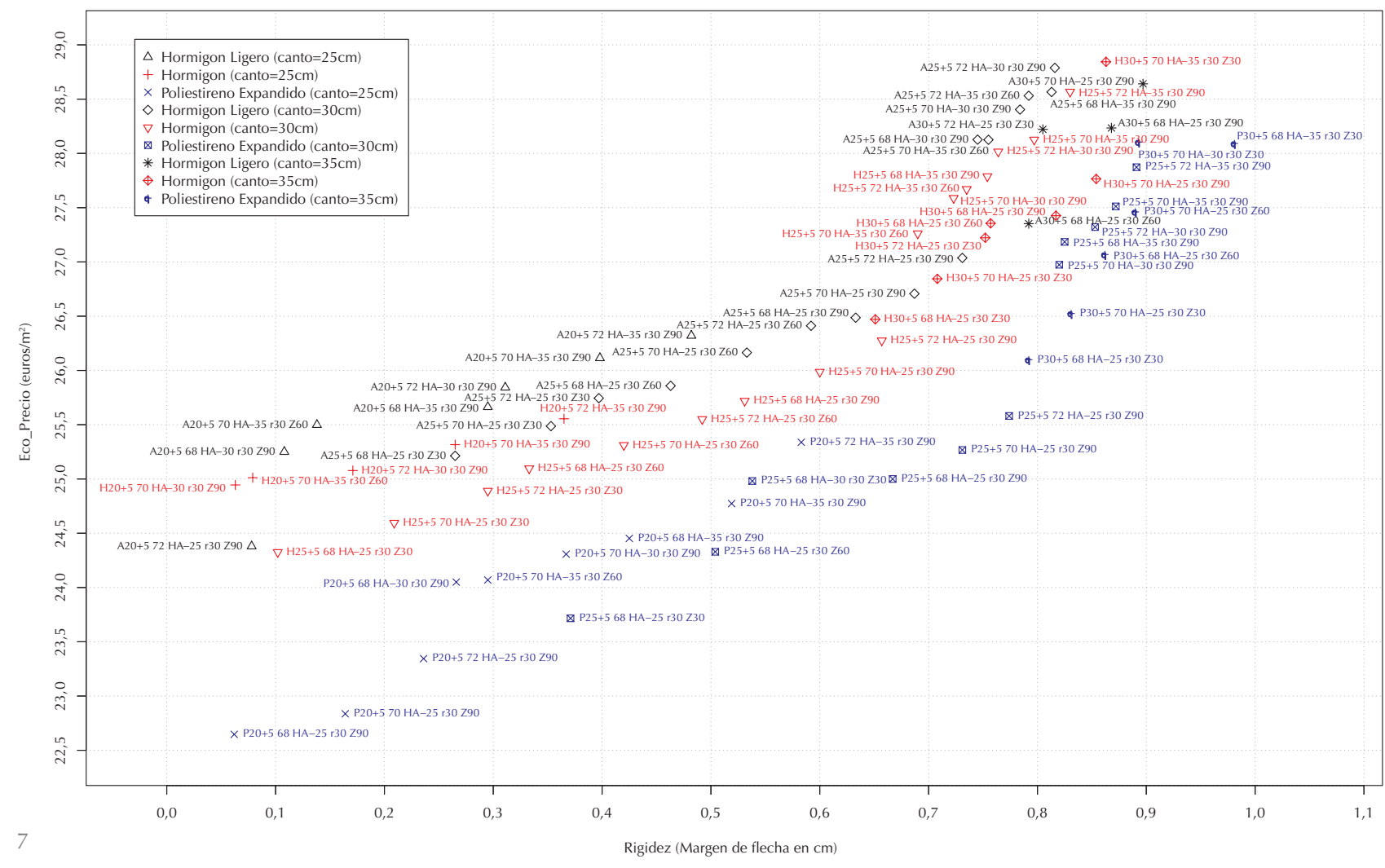

\section{RESULTADOS}

\subsection{Resultados para los forjados de 5 metros de luz}

En las Figuras 4 y 5 se muestran los óptimos de Pareto obtenidos para los forjados de 5 metros de luz con diversos cantos $(25,30$ y $35 \mathrm{~cm}$ ) y materiales aligerantes (hormigón, hormigón ligero y poliestireno expandido). La Figura 4 muestra las soluciones en base al coste material de ejecución medido en $€ / \mathrm{m}^{2}$ del forjado frente a la rigidez del mismo representada como la diferencia entre la flecha máxima admisible y la flecha estimada de cada solución.

Para reducir el número de casos y simplificar el gráfico, solamente se presentan aquellas soluciones que han tenido un coste menor que el coste medio de todos los óptimos de Pareto obtenidos para esa luz.

Con este gráfico, el diseñador puede identificar qué soluciones ofrecen mejor relación precio/rigidez frente a otras según los costes reales actualizados a la fecha de la toma de decisiones. En el caso de la Figura 4, aunque la utilización del poliestireno expandido es la elección que permite obtener mejores soluciones en cuanto a coste y rigidez, el diseñador puede valorar la diferencia en costes y rigidez que supondría la elección de otro tipo de material. De esta forma, puede elegir la mejor opción dentro de un amplio abanico de posibilidades y sin necesidad del procedimiento tradicional de prueba y error.

En este caso particular, las soluciones óptimas se consiguen con bovedillas de poliestireno expandido y canto de $25 \mathrm{~cm}$ (soluciones en azul y que comienzan con P20+5). La utilización de este material aporta soluciones con un consumo de ace$\mathrm{ro} / \mathrm{m}^{2}$ inferior a las de los otros materiales pues es un material ligero y de precio muy competitivo para este canto. Por el contrario, las soluciones encontradas para ese canto y en hormigón ligero (soluciones en negro y que comienzan con A20+5), tienen un sobrecoste que no se ve compensado con la disminución del consumo de acero debido a que los precios actuales de este material son muy elevados. Por último, las soluciones que utilizan bovedilla de hormigón como aligerante (soluciones en rojo que comienzan con $\mathrm{H} 20+5$ ) quedan en una zona intermedia.

También puede observarse que, a medida que se utilizan apoyos más anchos y por ser un canto pequeño, el aumento en el consumo de litros de hormigón por metro cuadrado aporta mayor rigidez de los apoyos mejorando las prestaciones del conjunto pero con un sobrecoste razonable.

La Figura 5, muestra las mismas soluciones pero empleando el ecoprecio en vez del 
8. Óptimos de Pareto para forjados de luz $=4 \mathrm{~m}$ (precio frente a rigidez).

9. Óptimos de Pareto para forjados de luz $=7 \mathrm{~m}$ (precio frente a rigidez). precio, donde se considera un futuro e hipotético escenario en el que existan gravámenes medioambientales. En este caso, de acuerdo con la ecuación (1) propuesta para el cálculo del ecoprecio y considerando un coste de $20 €$ por tonelada de $\mathrm{CO}_{2}$ emitida, las soluciones sufren un incremento medio del $1,50 \pm 0,04 € / \mathrm{m}^{2}$ para un intervalo de confianza del 95\% aunque, lógicamente, en un futuro este incremento dependerá del

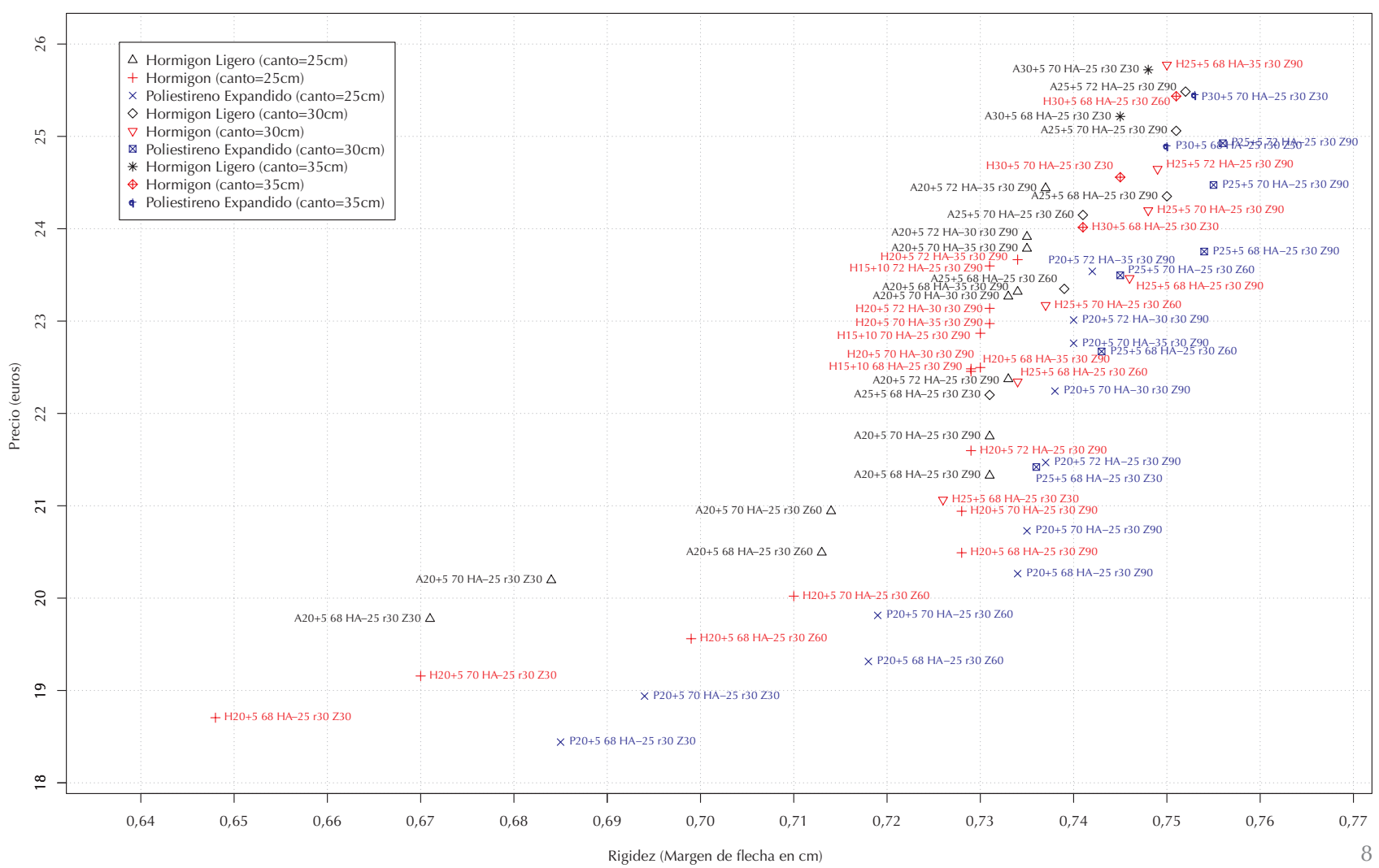

Gráfico de Soluciones Pareto para Luz de $7 \mathrm{~m}$ y Cantos de 30 y 35cm (Precio vs Rigidez)

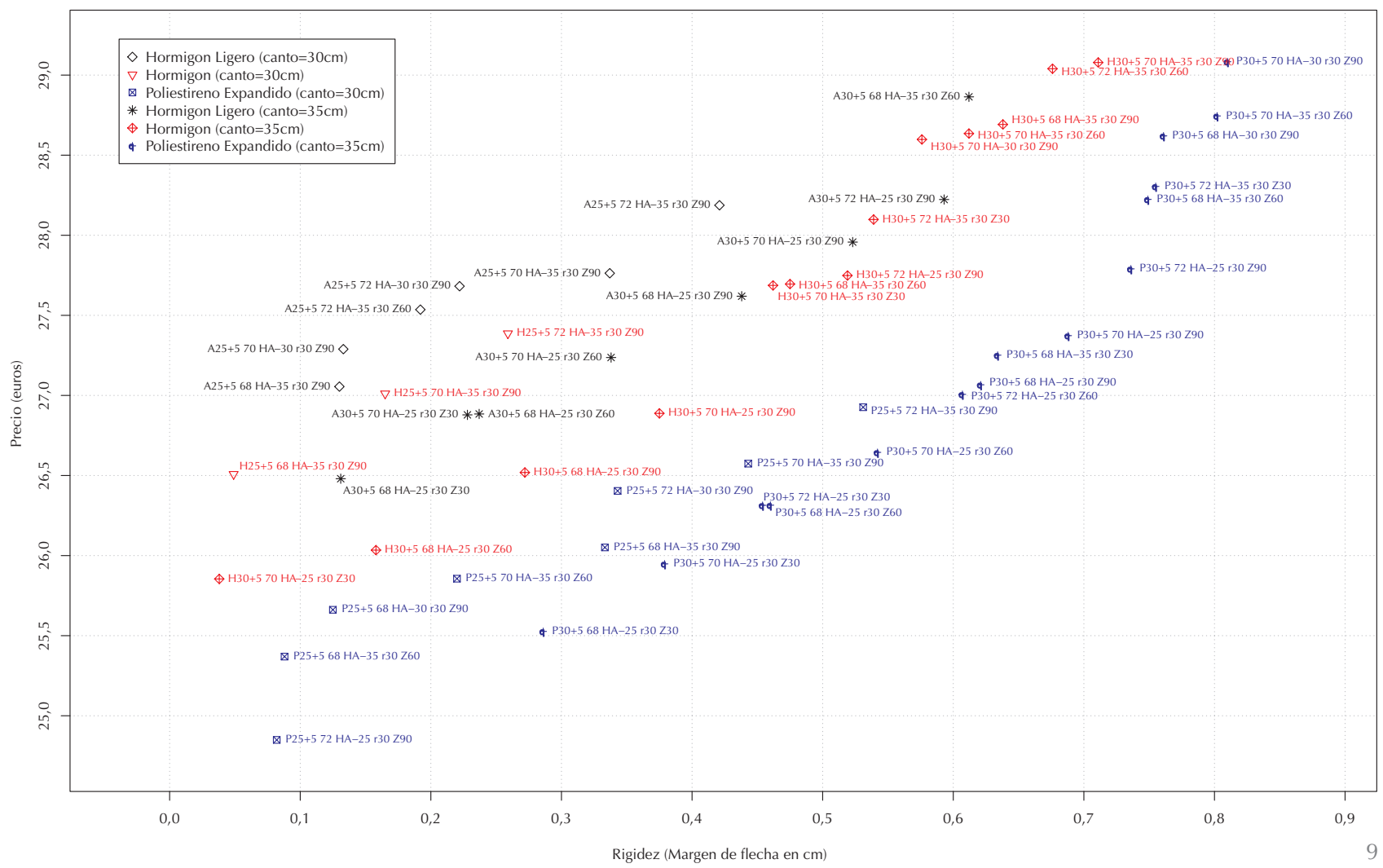


modo en que la legislación determine el cálculo de este gravamen.

Al considerar el ecoprecio y, según la simulación propuesta, las distancias entre los diferentes materiales se reducen ligeramente aunque las basadas en poliestireno expandido siguen siendo las que mejor relación "precio/prestaciones" presentan.

\subsection{Resultados para los forjados de 6 metros de luz}

En la Figura 6 se muestran los óptimos de Pareto para los forjados de 6 metros de luz y materiales y cantos estudiados.

La distribución de las soluciones según el material aligerante sigue el mismo esquema que en el caso de $5 \mathrm{~m}$ de luz donde el poliestireno expandido presenta las mejores soluciones frente al hormigón y hormigón ligero.

En cambio, se observa que a medida que se buscan soluciones con mayor margen de flecha o Rigidez, el canto de $30 \mathrm{~cm}$ mejora, en los tres materiales, a las prestaciones del canto de $25 \mathrm{~cm}$. Esto se produce fundamentalmente dentro del rango de rigidez de 0,35 a $0,80 \mathrm{~cm}$. A partir de este último valor las soluciones se disparan en cuanto precio.

La Figura 7 representa las soluciones de luz 6 m según el ecoprecio. En este caso, las diferencias entre los diferentes materiales se reducen ligeramente aunque la distribución es muy similar a la de la Figura 6. La repercusión obtenida al incluir las emisiones de $\mathrm{CO}_{2}$ representa un incremento medio de $1,53 \pm 0,03 € / \mathrm{m}^{2}$ para un intervalo de confianza del $95 \%$.

Finalmente, en las Figuras 8 y 9 , se muestran las soluciones para forjados de 4 y $7 \mathrm{~m}$ de luz y según el precio medio por metro cuadrado frente a la rigidez. Las soluciones con ecoprecio no se han incluido para no hacer demasiado extensivo el artículo, aunque la distribución de las soluciones en ellas son muy parecidas a las que corresponden con el precio.

\section{CONCLUSIONES}

En este artículo se ha presentado una metodología que permite la búsqueda de soluciones óptimas, equilibradas en coste y prestaciones, de forjados unidireccionales con nervio hormigonado in situ.

La idea principal consiste en realizar múltiples combinaciones de diseños obteniéndose, de todas aquellas soluciones válidas, el valor de la flecha que permite estimar el grado de bondad de la solución a efectos de rigidez y los consumos de los materiales. A partir de estos consumos se determina no solo el coste por metro cuadrado sino también un nuevo parámetro, denominado ecoprecio, que incorpora el coste debido a las emisiones de $\mathrm{CO}_{2}$.

A partir de los resultados, se obtienen los óptimos de Pareto según cada material, canto y luz; y se representan en un gráfico para un solo tipo de luz y según el precio o ecoprecio, y la Rigidez.

La utilización de este gráfico presenta múltiples ventajas al diseñador de forjados pues:

- Se presentan conjuntas las mejores soluciones para cada canto y cada material aligerante de modo que resulta fácil realizar comparaciones en cuanto a coste $y$ prestaciones.

- Se reduce el tiempo de diseño ya que se evita en gran medida el largo proceso de prueba y error.

Además, esta metodología, si se automatiza, puede permitir el análisis rápido de las soluciones óptimas en cada escenario futuro donde se produzcan cambios en los precios de los materiales, en los costes de mano de obra o la inclusión de futuros gravámenes como, por ejemplo, los debidos a las emisiones de $\mathrm{CO}_{2}$.

Finalmente, esta metodología se puede ampliar con la inclusión de otros parámetros que midan otras prestaciones del forjado como: comportamiento acústico, comportamiento térmico, resistencia al fuego, etc.

\section{REFERENCIAS}

(1) Alvarez-Ude, L. (2003). Edificación y desarrollo sostenible. GBC: Un método para la evaluación ambiental de edificios. Informes de la Construcción, 55(486): 63-70. doi:10.3989/ic.2003.v55.i486.556.

(2) Macías, M., García Navarro, J. (2010). Metodología y herramienta VERDE para la evaluación y la sostenibilidad en edificios. Informes de la Construcción, 62(517): 87-100. doi:10.3989/ic.08.056.

(3) Código Técnico de la Edificación. (2006, marzo). Ministerio de la Vivienda.

(4) Comisión Permanente del Hormigón. (2008). Instrucción de Hormigón Estructural EHE08. Ministerio de Fomento, Madrid. 
(5) Gómez-Hermoso, J. (1998). Análisis técnico económico de la influencia que presenta el empleo de diferentes materiales y tipologías estructurales en el proyecto de estructuras de edificios (Tesis doctoral). Madrid: Universidad Politécnica de Madrid.

(6) Payá, I., Yepes, V., Clemente, J.J., González, F. (2006). Optimización heurística de pórticos de edificación de hormigón armado. Revista internacional de Métodos Numéricos para Cálculo y Diseño en Ingeniería, 22(3): 241-259.

(7) Moragues-Terrades, J. (1980). Diseño óptimo de estructuras aporticadas de hormigón armado. (Tesis doctoral). Valencia: Universidad Politécnica de Valencia.

(8) Payá, I., Yepes, V., González, F., Hospitaler, A. (2008). Multiobjective Optimization of Concrete Building Frames by Simulated Annealing. Computer Aided Civil and Infrastructure Engineering, 23(8): 575-589.

(9) Castilho, V.C., El Debs, M.K., Nicoletti, M.C. (2007). Using a modified genetic algorithm to minimize the production costs for slabs of precast prestressed concrete joists. Engineering Applications of Artificial Intelligence, 20(4): 519-530.

(10) Payá, I., Yepes, V., Hospitaler, A., González, F. (2009). CO $\mathrm{CO}_{2}$-optimization of reinforced concrete frames by simulated annealing. Engineering Structures, 31(7): 1501-1508.

(11) CYPE Ingenieros S.A. (2011). CYPE Ingenieros Software para Arquitectura, Ingeniería y Construcción. Alicante.

(12) Instituto de Tecnología de la Construcción de Cataluña. (2011). Banco Estructurado de Datos de Elementos Constructivos BEDEC. Barcelona.

(13) Sistema electrónico de negociación de derechos de emisión de dióxido de carbono. http://www.sendeco2.com/.

(14) Ministerio de Fomento. (2010, febrero). Documentos Básicos. DB SE Seguridad Estructural. Código Técnico de la Edificación. Madrid. 